PROCEEDINGS OF THE

AMERICAN MATHEMATICAL SOCIETY

Volume 45, Number 2, August 1974

\title{
SOME FORMULAS IN THE STEENROD ALGEBRA
}

\author{
F. P. PETERSON 1
}

ABSTRACT. In this paper we prove that certain sums of Steenrod operations are zero. We also show how certain Milnor basis elements can be expanded in the Adem basis.

1. Introduction. The canonical anti-automorphism $\chi$ in the Steenrod algebra is defined by the equation $\sum_{i=0}^{k} \chi\left(\mathrm{Sq}^{i}\right) \mathrm{Sq}^{k-i}=0$ if $k>0$. For reasons unrelated to this paper, we wanted to know the value of $\sum_{i=0}^{k} \mathrm{Sq}^{i} \mathrm{Sq}^{k-i}$. Our study led to the results of this paper. Special cases of our main results (Theorems 3.1 and 4.1) show that

$$
\sum_{i=0}^{k} \mathrm{Sq}^{i} \mathrm{Sq}^{k-i}=0 \quad \text { if } k \neq 0(3)
$$

and, if $k \equiv 0(3)$,

$$
\sum_{i=0}^{k} \mathrm{Sq}^{i} \mathrm{Sq}^{k-i}=\mathrm{Sq}^{(0, k / 3)}=\sum_{i=2 k / 3}^{k} \mathrm{Sq}^{i} \mathrm{Sq}^{k-i} .
$$

2. $\operatorname{Hom}_{k}(A, A)$. Let $k$ be a field and let $A$ be a graded, connected, Hopf algebra over $k$. Let $H=\operatorname{Hom}_{k}(A, A)$ be all vector space maps $f$ of degree 0 such that $f_{0}: A_{0} \rightarrow A_{0}$ is $\eta_{0} \epsilon_{0}$. If $f, g \in H$, define $f * g \in H$ by $f * g=\phi(f \otimes g) \psi$. Let $0 \in H$ be the map which is 0 in positive dimensions and $\eta_{0} \epsilon_{0}$ in dimension 0 . The following propositions follow easily.

Proposition 2.1. $0 * f \doteq f * 0=f$.

Proposition 2.2. If $A$ is commutative or cocommutative, $f * g=g * f$.

Proposition 2.3. If $A$ is associative and coassociative, then $*$ is associative.

Proposition 2.4. If $f$ is an algebra map, then $f(g * h)=(f g) *(f h)$. If $f$ is a coalgebra map, then $(g * h) f=(g f) *(h f)$.

Received by the editors December 22, 1972 and, in revised form, March 15, 1973. AMS (MOS) subject classifications (1970). Primary 55G10.

Key words and phrases. Steenrod algebra, Milnor basis, Adem basis.

1 The author was partially supported by the N.S.F. 
Proposition 2.5. If $f$ and $g$ are algebra maps and $A$ is commutative, then $f * g$ is an algebra map. If $f$ and $g$ are coalgebra maps and $A$ is cocommutative, then $f * g$ is a coalgebra map.

Proposition 2.6. Under the assumptions of Propositions 2.2 and 2.3, $\operatorname{Hom}_{k}(A, A)$ is an abelian group under *.

3. The case $A$ is the Steenrod algebra. As an example of some of these notions, we look at the case when $A=\Theta_{p}$, the $\bmod p$ Steenrod algebra. Let id $\epsilon H$ denote the identity map, and define $t_{r} \in H$ by $t_{1}=$ id and $t_{r}=$ $t_{r-1} *$ id. For example, $t_{2}=\mathrm{id} *$ id and

$$
t_{2}\left(\mathrm{Sq}^{k}\right)=\sum_{i=0}^{k} \mathrm{Sq}^{i} \mathrm{Sq}^{k-i} \text { if } p=2 .
$$

Let $\mathrm{Sq}^{R}$ and $\mathcal{P R}^{R}$ be the Milnor basis elements [2] corresponding to sequences $R=\left(r_{1}, \cdots\right)$. Let $\mathrm{Sq}_{r}(k)$ and $\mathcal{P}_{r}(k)$ be the elements corresponding to the sequence $(0, \cdots, 0, k, 0, \cdots)$, with the nonzero term in the $r$ th place. Let $p_{k}=1+p+\cdots+p^{k-1}$. Let $\mathcal{P}=1+\mathfrak{P}^{1}+\mathcal{P}^{2}+\cdots$ If $S=\left(s_{1}, \cdots\right)$ is a Milnor basis sequence, define $\left(\begin{array}{c}r \\ S\end{array}\right)=\left(\begin{array}{c}r \\ 1\end{array}\right)^{s} 1\left(\begin{array}{c}r \\ 2\end{array}\right)^{s} \ldots$, with the convention that $(0)^{0}$ $=1$.

Theorem 3.1. $t_{r}(\mathcal{P})=\Sigma_{S}\left(\begin{array}{l}r \\ S\end{array}\right) \mathcal{P}$.

Corollary 3.2. If $p=2, t_{2}(\mathrm{Sq})=\Sigma_{k=0}^{\infty} \mathrm{Sq}_{2}(k)$.

In order to prove Theorem 3.1, we study $t_{r}^{*}: \mathbb{P}^{*} \rightarrow \mathbb{Q}^{*}$, the dual map. This is a map of algebras by Proposition 2.5. Let $I \subset \mathbb{Q}^{*}$ be the ideal generated by $\xi_{2}, \xi_{3}, \cdots, \tau_{0}, \tau_{1}, \ldots$. We first prove the following lemma.

Lemma 3.3. $t_{r}^{*}\left(\xi_{k}\right) \equiv\left(\begin{array}{c}r \\ k\end{array}\right)\left(\xi_{1}\right)^{p_{k}} \bmod I$ if $k>0 . t_{r}^{*}\left(\tau_{k}\right) \equiv 0 \bmod I$ if $k \geq 0$.

Proof. We prove this by induction on $r_{0} t_{1}^{*}$ is the identity and the result is true. In general,

$$
\begin{aligned}
t_{r}^{*}\left(\xi_{k}\right) & =\sum_{i=0}^{k} t_{r-1}^{*}\left(\xi_{k-i}\right)^{p^{i}} \xi_{i} \equiv t_{r-1}^{*}\left(\xi_{k}\right) \xi_{0}+t_{r-1}^{*}\left(\xi_{k-1}\right)^{p} \xi_{1} \bmod I \\
& \equiv\left(\begin{array}{c}
r-1 \\
k
\end{array}\right)\left(\xi_{1}\right)^{p} k+\left(\begin{array}{c}
r-1 \\
k-1
\end{array}\right)^{p}\left(\xi_{1}\right)^{p \cdot p} k-1+1 \bmod I \\
& =\left(\begin{array}{c}
r \\
k
\end{array}\right)\left(\xi_{1}\right)^{p} k \bmod I .
\end{aligned}
$$


Also, $t_{r}^{*}\left(\tau_{k}\right) \equiv 0 \bmod I$ as it is odd dimensional.

Proof of 3.1. Let $m=\xi_{1}^{s_{1}} \cdots \xi_{k}^{s_{k}} \in \mathbb{P}^{*}$. Then $\left\langle t_{r}(\mathcal{P}), m\right\rangle=\left\langle\mathcal{P}, t_{r}^{*}(m)\right\rangle$ $=\left(\begin{array}{c}r \\ 1\end{array}\right)^{s} \ldots\left(\begin{array}{c}r \\ k\end{array}\right)^{s k}$ by Lemma 3.3. If $m$ involves one of the $\tau^{\prime} s,\left\langle t_{r}(\mathscr{P}), m\right\rangle=$ 0 . This proves Theorem 3.1.

4. $\mathcal{P}(0, k)$. Motivated by Corollary 3.2 and the fact that $\mathscr{P}_{r}(k)$ plays an important role in the study of modules over the Steenrod algebra (see [1] and [3]), we will show how $\mathcal{P}(0, k)$ can be expanded in the Adem basis for $\mathbb{Q}_{p}$.

Theorem 4.1. $\mathscr{P}(0, k)=\sum_{s=0}^{k}(-1)^{s+k} \mathcal{P}(p+1) k-s \mathcal{P} s=$ the signed sum of all admissible Adem basis elements of length $\leq 2$ and of degree $k(p+1)(2 p-2)$.

Before proving Theorem 4.1, we need a couple of lemmas and a corollary.

Lemma 4.2. $\sum_{t=0}^{m}(-1)^{t+1\left(m_{t}+f\right)}=(-1)^{m+1(\underset{m}{m+f-1})}$ if $f>0$.

Lemma 4.3. $(\underset{m}{(p+1) m-1)} \equiv 0(p)$.

Corollary 4.4. $\Sigma_{t=0}^{m}(-1)^{t+1}\left(\left(\begin{array}{c}p+1) m \\ t\end{array}\right) \equiv 0(p)\right.$.

Proof of 4.2. $\Sigma_{t=0}^{m}(-1)^{t+1}\left(\begin{array}{c}m+f \\ t\end{array}\right)=\Sigma_{t=0}^{m}(-1)^{t+1}\left[\left(\begin{array}{c}m+f-1 \\ t\end{array}\right)+\left(\begin{array}{c}m+f-1 \\ t-1\end{array}\right)\right]=$ $\left.(-1)^{m+1} \underset{m}{(m+f-1}\right)$ by telescoping.

Proof of 4.3. Let $m=b_{0} p^{a}+b_{1} p^{a+1}+\cdots, 0<b_{0}<p$, be the $p$-adic expansion of $m$. Then $(p+1) m=b_{0} p^{a}+\left(b_{1}+1\right) p^{a+1}+\cdots$ and $(p+1) m-1$ $=(p-1)+(p-1) p+\cdots+(p-1) p^{a-1}+\left(b_{0}-1\right) p^{a}+\left(b_{1}+1\right) p^{a+1}+\cdots$ and hence $\left(\underset{m}{(p+1) m-1)} \equiv 0(p)\right.$ as the term corresponding to $p^{a}$ is

$$
\left(\begin{array}{c}
b_{0}-1 \\
b_{0}
\end{array}\right) \equiv 0(p)
$$

Proof of 4.4. Corollary 4.4 follows from Lemmas 4.2 and 4.3 by taking $f=p m$.

We now prove Theorem 4.1. We use Milnor's theorem [2] that

$$
\mathcal{P} t \mathcal{P}^{s}=\sum_{i=0}^{s}\left(\begin{array}{c}
t+s-(p+1) i \\
s-i
\end{array}\right) \mathcal{P}^{(t+s-(p+1) i, i)} .
$$

Hence, 


$$
\begin{aligned}
& \sum_{s=0}^{k}(-1)^{s} \mathcal{P}^{(p+1) k-s \rho s}=\sum_{s=0}^{k} \sum_{i=0}^{s}(-1)^{s}\left(\begin{array}{c}
(p+1)(k-i) \\
s-i
\end{array}\right) \mathcal{P ( ( p + 1 ) ( k - i ) , i )} \\
& =\sum_{i=0}^{k-1}\left(\sum_{s=i}^{k}(-1)^{s}\left(\begin{array}{c}
(p+1)(k-i) \\
s-i
\end{array}\right)\right) \mathfrak{P}^{((p+1)(k-i), i)}+(-1)^{k} \mathcal{P}(0, k) \text {. }
\end{aligned}
$$

But

$$
\sum_{s=i}^{k}(-1)^{s}\left(\begin{array}{c}
(p+1)(k-i) \\
s-i
\end{array}\right)=(-1)^{i-1} \sum_{t=0}^{m}(-1)^{t+1}\left(\begin{array}{c}
(p+1) m \\
t
\end{array}\right) \equiv 0(p)
$$

by Corollary 4.4. Hence the theorem is proved.

Corollary 4.5. If $k \neq 0(3)$, then $\sum_{i=0}^{k} \mathrm{Sq}^{i} \mathrm{Sq}^{k-i}=0$. If $k \equiv 0(3)$, then $\sum_{i=0}^{2 k / 3-1} \mathrm{Sq}^{i} \mathrm{Sq}^{k-i}=0$.

Proof. The first part follows directly from Corollary 3.2 and the second part follows from Corollary 3.2 and Theorem 4.1.

Theorem 4.1 might lead one to conjecture that $\mathcal{P}_{r}(k)=$ the signed sum of all admissible Adem basis elements of length $\leq r$ and of degree $p_{r} k$. Calculation shows that this is true for $(k=1, r=3, p=2)$, but false for $(k=2$ or $3, r=3, p=2),(k=1, r=3, p=3)$, and $(k=1, r=4, p=2)$.

\section{BIB LIOGR APHY}

1. J. F. Adams and H. R. Margolis, Modules over the Steenrod algebra, Topology 10 (1971), 271-282. MR $45 \# 3520$.

2. J. W. Milnor, The Steenrod algebra and its dual, Ann. of Math. (2) 67 (1958), 150-171. MR $20 \# 6092$.

3. J. C. Moore and F. P. Peterson, Modules over the Steenrod algebra, Topology 11 (1972), 387-395.

DEPARTMENT OF MATHEMATICS, MASSACHUSETTS INSTITUTE OF TECHNOLOGY, CAMBRIDGE, MASSACHUSETTS 02139 